\title{
An Energy Minimization Reconstruction Algorithm for Multivalued Discrete Tomography*
}

\author{
László Varga \& Péter Balázs \& Antal Nagy \\ Department of Image Processing and Computer Graphics \\ University of Szeged \\ Árpád tér 2, H-6720 Szeged, Hungary \\ \{vargalg, pbalazs, nagya\}@inf.u-szeged.hu
}

We propose a new algorithm for multivalued discrete tomography, that reconstructs images from few projections by approximating the minimum of a suitably constructed energy function with a deterministic optimization method. We also compare the proposed algorithm to other reconstruction techniques on software phantom images, in order to prove its applicability.

Key words: multivalued discrete tomography; reconstruction; GPGPU; optimization; non-destructive testing

\section{Introduction}

Tomography deals with the reconstruction of objects from a given set of their projections. This is usually done by exposing the object to some electromagnetic or particle radiation, and measuring the loss of the energy as the beams pass through it. With this information one can derive the integrals of attenuation coefficients along the path of the beams, and obtain the inner structure of the object.

There are several suitable algorithms for tomography, which can provide satisfactory reconstructions of arbitrary objects, when a sufficiently high amount of information (which usually means hundreds of projections) is available (12).

In discrete tomography (DT) $(10 ; 11)$, one assumes that the object to be reconstructed consists of only a few different materials with known attenuation co-

* This research was in part supported by the TÁMOP-4.2.1/B09/1/KONV-2010-0005 project of the Hungarian National Development Agency co-financed by the European Union and the European Regional Development Fund. The work of the second author was also supported by the János Bolyai Research Scholarship of the Hungarian Academy of Sciences and the PD100950 grant of the Hungarian Scientific Research Fund (OTKA).

${ }^{\dagger}$ The publication is supported by the European Union and cofunded by the European Social Fund. Project title: "Broadening the knowledge base and supporting the long term professional sustainability of the Research University Centre of Excellence at the University of Szeged by ensuring the rising generation of excellent scientists." Project number: TÁMOP-4.2.2/B-10/1-20100012 efficients. Binary tomography - as a special case of DT - makes the additional restriction that the reconstructed volume contains only two materials. With such prior information, the reconstruction can be performed even from a few projections. DT can be particularly useful, e.g., in non-destructive testing (6), where the goal is to gain some information of the interior of - usually homogeneous - objects without damaging them.

There is a wide range of algorithms for binary and non-binary (called multivalued) discrete tomography. For example, the DART, Discrete Algebraic Reconstruction Technique (4) is capable of producing highly accurate reconstructions by thresholding a continuous reconstruction and then adjusting the object boundaries. Also, there are reconstruction algorithms based on minimizing an energy function by deterministic $(13 ; 15 ; 16 ; 18)$ or randomized $(1 ; 2 ; 8 ; 14)$ optimization strategies.

In this paper we propose a deterministic reconstruction method for multivalued discrete tomography, that solves the problem by minimizing a suitably constructed energy function. The basic idea behind our new method was provided by the algorithm given in (15), that is a highly accurate binary reconstruction algorithm based on D.C. programming - a method for minimizing the difference of convex functions.

Unfortunately, the DC algorithm is restricted to the reconstruction of binary images. Our goal is to provide a valuable extension, that is suited for the general 
case of multivalued DT. Although other simple extensions of the DC algorithm also exist (see, e.g., (13; $16 ; 18)$ ), we propose significant modifications of the original method, to supply an algorithm that is fully adjusted to multivalued DT. We introduce a new energy function for modeling the possible values of the reconstruction, and we also define a new process that can perform a fast approximate optimization of the energy function.

The paper is structured as follows. In Section 2 we give a brief description of the theoretical background of discrete tomography. Then, in Section 3 we describe the proposed method, and in Section 4 we provide experimental results. Finally, in Section 5 we summarize the results.

\section{Discrete Tomography}

For a simple formalism we present our reconstruction algorithm in the case of two-dimensional tomography, but the method can easily be extended to higher dimensions, too. The model we use assumes that a single slice of the reconstructed object is represented by an $n \times n$ size digital image. Moreover, we assume parallel beam projection geometry, i.e., a projection is given by projection values corresponding to parallel projection rays, where each value is given by the integral of the image on a straight line.

With the above considerations the discrete reconstruction problem can be represented by a system of equations

$$
\mathbf{A x}=\mathbf{b}, \quad \mathbf{A} \in \mathbb{R}^{m \times n^{2}}, \mathbf{x} \in L^{n^{2}}, \mathbf{b} \in \mathbb{R}^{m},
$$

where

- $\mathbf{x}$ is the vector of all $n^{2}$ unknown image pixels,

- $m$ is the total number of projection lines used,

- b is the vector of all $m$ measured projection values,

- A describes the projection geometry with all $a_{i j}$ elements giving the length of the line segment of the $i$-th projection line through the $j$-th pixel,

- and $L=\left\{l_{0}, l_{1}, \ldots, l_{c}\right\}$ is the set of the possible intensities (assuming that $l_{0}<l_{1}<\ldots<l_{c}$ ).

An illustration of the applied projection geometry can be seen in Fig. 1.

Note that - as a special case - with $L=\{0,1\}$ we arrive to the well-known model of binary tomography.

With the above formulation the reconstruction is equivalent to the task of solving the equation system given in (1). Unfortunately, beside the problems arising from the fact that we search a discrete-valued solution, the system of (1) is usually extremely huge,
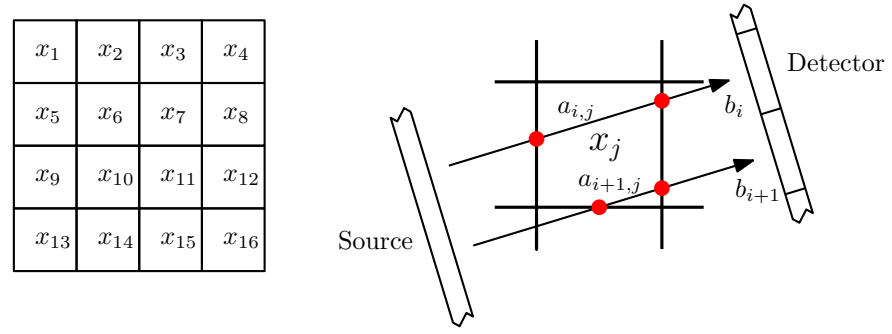

Figure 1: Representation of the parallel beam geometry on a discrete image.

and often underdetermined (owing to the low number of projections) or inconsistent (due to measurement errors). Various techniques have been suggested to overcome these problems, but all of them are heuristic methods. Efficient exact reconstruction algorithms exist only for some special classes of (mostly binary) images (see, e.g., (5; 7)).

\section{The Proposed Method}

Since, even in the binary case, the discrete reconstruction problem is NP-hard if the number of projections is more than two (9), our aim is to provide an approximate solution of the reconstruction task. The algorithm we propose performs the discrete reconstruction by minimizing a suitably constructed energy function.

\subsection{The Energy Function}

The energy function consists of two terms. Using the notation of Sect. 2 it can be given as

$$
\mathcal{E}_{\mu}(\mathbf{x}):=f(\mathbf{x})+\mu \cdot g(\mathbf{x}), \quad \mathbf{x} \in\left[l_{0}, l_{c}\right]^{n^{2}} .
$$

In more detail, the first function

$$
f(\mathbf{x})=\frac{1}{2} \cdot\|\mathbf{A x}-\mathbf{b}\|_{2}^{2}+\frac{\alpha}{2} \cdot \mathbf{x}^{T} \mathbf{S} \mathbf{x}
$$

is a formulation of the continuous reconstruction problem, where $\mathbf{S}$ is a matrix such that

$$
\mathbf{x}^{T} \mathbf{S} \mathbf{x}=\sum_{i=1}^{n^{2}} \sum_{j \in N_{4}(i)}\left(x_{i}-x_{j}\right)^{2}
$$

and $N_{4}(i)$ is the set of pixel indexes 4-connected to the $i$-th pixel.

Informally, $f(\mathbf{x})$ consists of an $\|\mathbf{A x}-\mathbf{b}\|_{2}^{2}$ projection correctness (or data fidelity) term, and an $\mathbf{x}^{T} \mathbf{S x}$ smoothness prior, that is lower if the reconstructed image is smooth, and thus it forces the results to contain larger homogeneous regions.

The second, $\mu \cdot g(\mathbf{x})$, term of (2) is a formulation of the discreteness, which propagates solutions containing values only from the $L$ predefined set of intensities. Here, $\mu \geq 0$ is a constant weight that can be used 


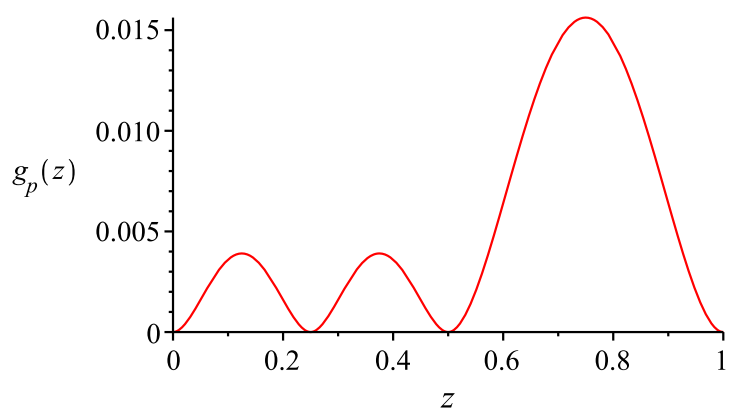

Figure 2: Example of the $g_{p}(z)$ one-variable discretization function with intensity values $L=$ $\{0,0.25,0.5,1\}$.

to balance between the two separate parts of the energy function, and $g(\mathbf{x})$ is constructed to take its minimal values at discrete solutions (i.e., when $\mathbf{x} \in L^{n^{2}}$ ) and higher positive values otherwise. The $g(\mathbf{x})$ discretizing function is given in the form

$$
g(\mathbf{x})=\sum_{i=1}^{n^{2}} g_{p}\left(x_{i}\right), \quad i \in\left\{1,2, \ldots, n^{2}\right\},
$$

where $g_{p}$ is a one-variable function composed of a set of forth-grade polynomial functions defined over the intervals of $L$ in the way

$g_{p}(z)= \begin{cases}\frac{\left[\left(z-l_{j-1}\right) \cdot\left(z-l_{j}\right)\right]^{2}}{2 \cdot\left(l_{j}-l_{j-1}\right)^{2}}, & \text { if } z \in\left[l_{j-1}, l_{j}\right] \text { for } \\ \text { undefined, } & \text { each } j \in\{1, \ldots, c\} \\ \text { otherwise. }\end{cases}$

An illustration of a $g_{p}$ function can be seen in Fig. 2 . Informally, this discretization function assigns a small value to each pixel if the pixel value in the reconstruction is close to an element of $L$, and higher values (increasing with the distance) otherwise. There are several other possible functions which could be used for such purposes (see, e.g., $(13 ; 16 ; 18))$. We have decided to construct this novel one, since it is easy to handle and can be efficiently computed.

\subsection{The Optimization Process}

The process of the optimization in our proposed method is based on breaking the energy function (2) into two parts, and prioritizing between them. The first part is given by the $f(\mathbf{x})$ defined in (3), i.e., two terms responsible for projection correctness and smoothness. The other part is provided by the $\mu \cdot g(\mathbf{x})$ discretization term.

In the beginning, the reconstruction algorithm assumes that the first two terms in the energy function prioritizes the discretization term. Therefore, the process will first focus on finding a continuous reconstruction, and neglect the discretization term. Afterwards, when a good approximation of the continuous reconstruction is found, the weight of the discretization term will be increased, thus the optimization process is steered towards a discrete solution.

The description of the algorithm uses the following notations.

- A, b, $\mathbf{x}$ and $n$ are as defined in Sect. 2,

- $\nabla g_{p}\left(x_{i}\right)$ denotes the derivate of the discretization term applied for the $i$-th $x_{i}$ pixel of the reconstructed image,

$$
\begin{aligned}
\nabla g_{p}(z)= & \frac{\left(z-l_{j-1}\right)\left(z-l_{j}\right)\left(2 \cdot z-l_{j-1}-l_{j}\right)}{\left(l_{j}-l_{j-1}\right)^{2}} \\
& \text { if } z \in\left[l_{j-1}, l_{j}\right]
\end{aligned}
$$

- $G_{0, \sigma}(z)$ is an unnormalized Gaussian function with 0 mean and $\sigma$ deviance, that is

$$
G_{0, \sigma}(z)=e^{-\left(\frac{z^{2}}{2 \cdot \sigma^{2}}\right)}
$$

- $\alpha \geq 0, \mu \geq 0$, and $\sigma \geq 0$ are predefined constants controlling in the energy function, respectively, the weight of the smoothness term, the weight of the discretization term, and the deviance of the Gaussian function applying the adaptive weighting of the discretization,

- $\lambda$ is an upper bound of the largest eigenvalue of the matrix $\left(\mathbf{A}^{T} \mathbf{A}+\alpha \cdot \mathbf{S}\right)$, that is used for reasons described in (15).

For obtaining the result, the optimization method uses an adaptive and automatic pixel-based weighting of the discretization term. The detailed description of the algorithm is given in Algorithm 1.

The optimization process makes a connection between the two parts of the energy function (i.e., the formulation of the continuous reconstruction problem, and the discretization term), and assumes that the first part has a higher priority (as our first consideration is to find a reconstruction that satisfies the projections, but we would also like to get a discrete result if possible).

With this, the algorithm is based on optimizing the energy function with a simple projected subgradient method, while applying an automatic weighting between the two terms of the energy function. In each iteration step of the optimization process, one can calculate the gradient of the $\|\mathbf{A x}-\mathbf{b}\|_{2}^{2}$ projection correctness term in the energy function by computing the $\mathbf{A}^{T}(\mathbf{A x}-\mathbf{b})$ vector. For each pixel, this vector explicitly contains an estimation of correctness of the pixel in the current solution according to the projections (the higher this value is the more responsible the 


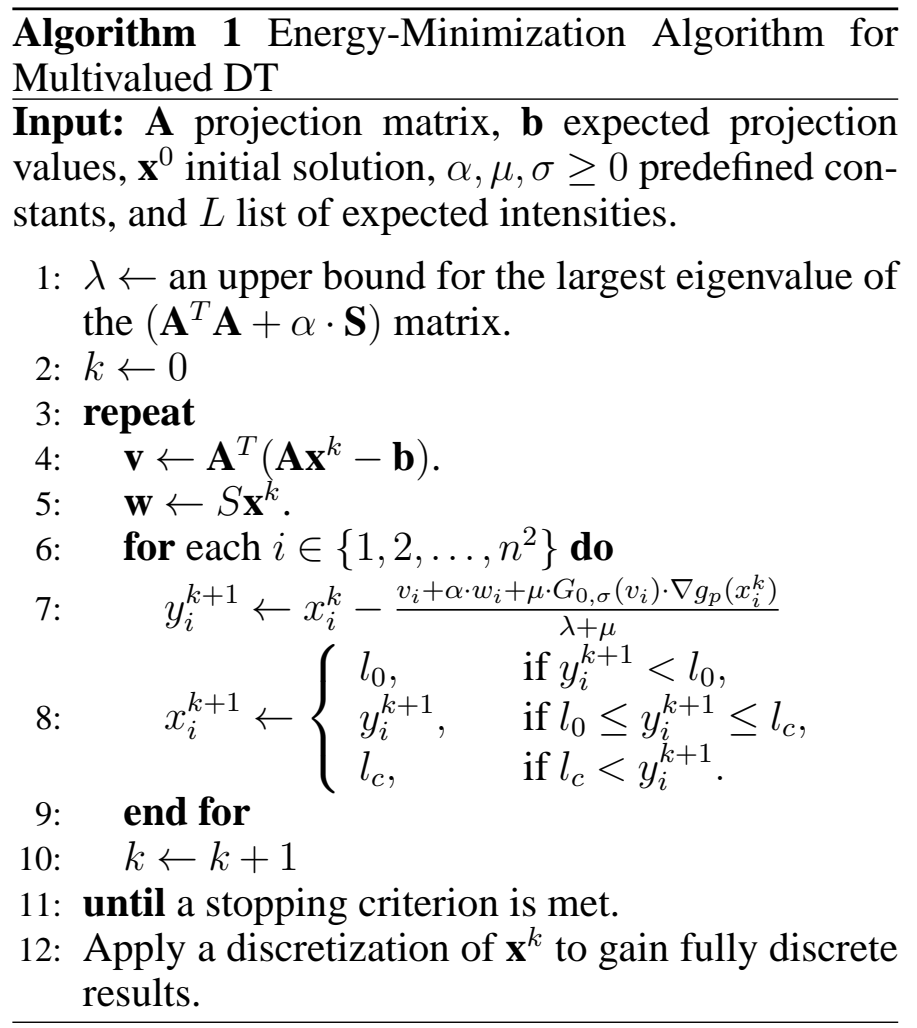

pixel is for causing incorrect projections). If we apply a Gaussian function on these values we can get a weight, that is smaller when the corresponding pixel needs further adjustments, and higher if the projection rays connected to that specific pixel are more or less satisfied. By weighting the discretization with this value calculated from the gradient of the projection correctness, one can apply an automatic adjustment of the discretizing term for each pixel, omitting it when the projections are not satisfied, and slowly increasing its effect as the pixel values get closer to an acceptable reconstruction.

In practice this means that the method starts with an arbitrary initial solution, and first approximates a continuous reconstruction based on the given set of projections. Later, as the projections of the solution get closer to the described vectors, the automatic weighting of the discretizing term begins to increase for each pixel. Thus the pixels will be slowly steered towards discrete values of $L$.

It is possible that the process will get stuck in a local minimum of the energy function. In this case the process will stop in a semi-continuous solution, where some pixels are properly discretized, and the rest of them are left continuous, since the projection correctness did not allow a full discretization. The $\mu$ and $\sigma$ parameters, are used to control the maximal strength of the discretizing term, and the speed at which the discretizing term gets strengthened during the process, respectively.

Finally, after the optimization process we complete the discretization by simply thresholding the pixel values, to gain a fully discrete reconstruction result.

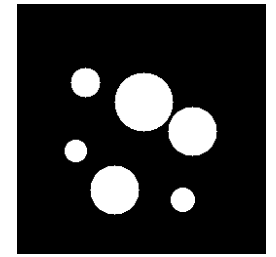

a)

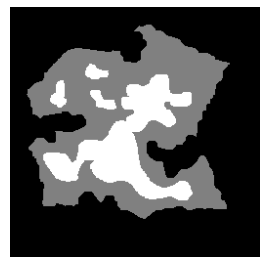

b)

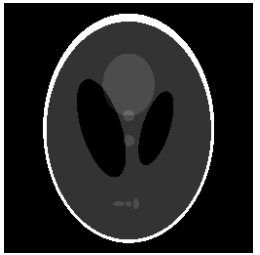

c)
Figure 3: Some of the software phantoms used for testing. a) a binary image; b) a multivalued image from (4); c) the well-known Shepp-Logan head phantom (see, e.g., page 53 of (12)).

The final thresholding of the result can be performed with values chosen half-way between neighboring intensity levels as

$$
x_{i}= \begin{cases}l_{0}, & \text { if } x_{i}^{k}<\left(l_{0}+l_{1}\right) / 2 \\ l_{j}, & \text { if }\left(l_{j-1}+l_{j}\right) / 2 \leq x_{i}^{k}<\left(l_{j}+l_{j+1}\right) / 2 \\ l_{c}, & \text { if }\left(l_{c-1}+l_{c}\right) / 2 \leq x_{i}^{k}\end{cases}
$$

where $\mathbf{x}^{k}$ is the result of the iterative optimization process of Alg. $1, j \in\{1, \ldots, c-1\}$, and $i$ takes each element of the set $\left\{1, \ldots, n^{2}\right\}$ as a value.

\section{Experimental Results}

We conducted experiments to compare our method to other published algorithms. On one hand, on binary images, we compared our new method to the DC algorithm, to see how the original, and our new approach performs related to each other. Unfortunately, due to the limitations of the DC algorithm (as it is not suited for multivalued tomography), we could only do this comparison for binary images. Also, we ran tests with the recently published DART (4) in order to compare the reconstruction of multivalued images.

We performed the evaluations, by using a set of phantom images (all having a size of 256 by 256 pixels). Three of these phantoms can be seen in Fig. 3. The reconstructions were performed from projection sets containing 2 to 18 projections, distributed equiangularly on the half circle, assuming that the projection with $0^{\circ}$ angle corresponds to vertical rays. The angle sets describing the projection directions for a $p$ projection number can be given as

$$
S(p)=\left\{i \cdot \frac{180^{\circ}}{p} \mid i=0, \ldots, p-1\right\} .
$$

As mentioned above, we used a parallel beam projection geometry, where projection values were given by line integrals on the image. The distances between neighboring projection lines were set to be one unit (the width of one pixel on the image), the rotation center of the projections was located in the center of the image half way between two projection lines, and in each projection the rays covered the whole image. 
In our tests, the parameters of the DART and DC algorithms were mostly set from the literature, with slight adjustments to get the best performance of all the methods in our tests. The parameters of the DC algorithm were set as given in (17) except that the strength of the smoothness term was $\alpha=2.5$. In DART, we used 10 iterations of the Simultaneous Iterative Reconstruction Technique (see, e.g., (12)) for performing the continuous reconstructions, applied the same smoothing kernel as described in (3), and terminated the algorithm when the thresholded image did not change in the last 10 DART iterations or the number of iterations reached a limit of 500 .

For the parameters of the proposed method, we used the values $\alpha=2.5, \mu=20, \sigma=1$, and in the $\mathbf{x}^{0}$ initial solution all the $x_{i}^{0}$ positions were set to the same value in the middle of the range of possible intensities (i.e., $x_{i}^{0}=\left(l_{c}-l_{0}\right) / 2$, for all $i \in\left\{1, \ldots, n^{2}\right\}$ ). The iteration was stopped when the difference between the solutions of the $k$-th and $(k+1)$-th iteration steps computed as $\left\|\mathbf{x}^{k+1}-\mathbf{x}^{k}\right\|_{2}$ became less then 0.001 or the number of iterations reached a limit of 5000. Although, the convergence of the optimization process is not yet proven, we found the algorithm to be convergent in all our tests with these parameter settings.

We implemented the algorithms in $\mathrm{C}++$ with GPU acceleration using the NVIDIA CUDA $\mathrm{C}$ sdk. The computation was performed on a PC, with an Intel Q9500 CPU, and an NVIDIA Geforce GTS250 GPU.

After reconstructing the results using all three algorithms, we compared them visually, and by using the error measurement

$$
E r r=\frac{D\left(\mathbf{x}, \mathbf{x}^{*}\right)}{O\left(\mathbf{x}^{*}\right)} \cdot 100 \%,
$$

where $D\left(\mathbf{x}, \mathbf{x}^{*}\right)$ is the number of misclassified pixels on the result, and $O\left(\mathbf{x}^{*}\right)$ is the number of non-zero pixels on the original phantom.

In addition, we also measured the computation times of the algorithms in each case. A summary of the numerical results can be seen in Table 4, while Fig. 4 and Fig. 5 give some examples of the reconstructed results of binary and multivalued images.

Based on the results we can deduce the following. In case of using very few projections (i.e., 2-3 projections for simple images like the phantoms of figures 3a-b, and up to 5-6 projections for more complex ones like Figure 3c), there was obviously not enough information for the reconstruction algorithms to give accurate solutions. Usually DART produced the best results, but this seems to be irrelevant since the reconstruction error is unacceptably high.

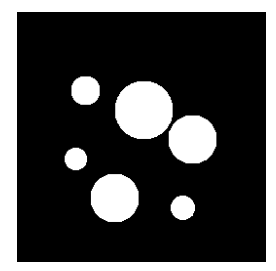

DC,

5 proj.

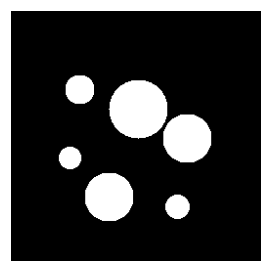

$\mathrm{DC}$

6 proj.

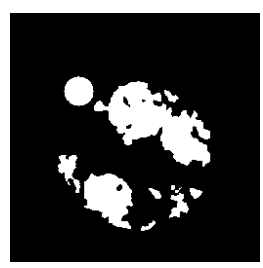

DART,

5 proj.

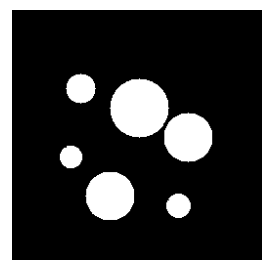

DART,

6 proj.

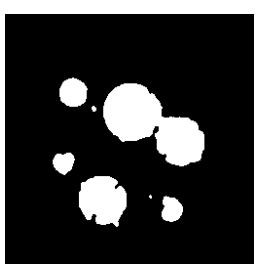

Prop. meth., 5 proj

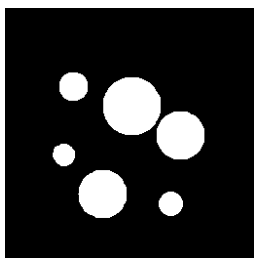

Prop. meth., 6 proj
Figure 4: Reconstructions of a binary phantom (Figure $3 \mathrm{a}$ ), produced by the three compared algorithms, from projection sets containing different numbers of projections.

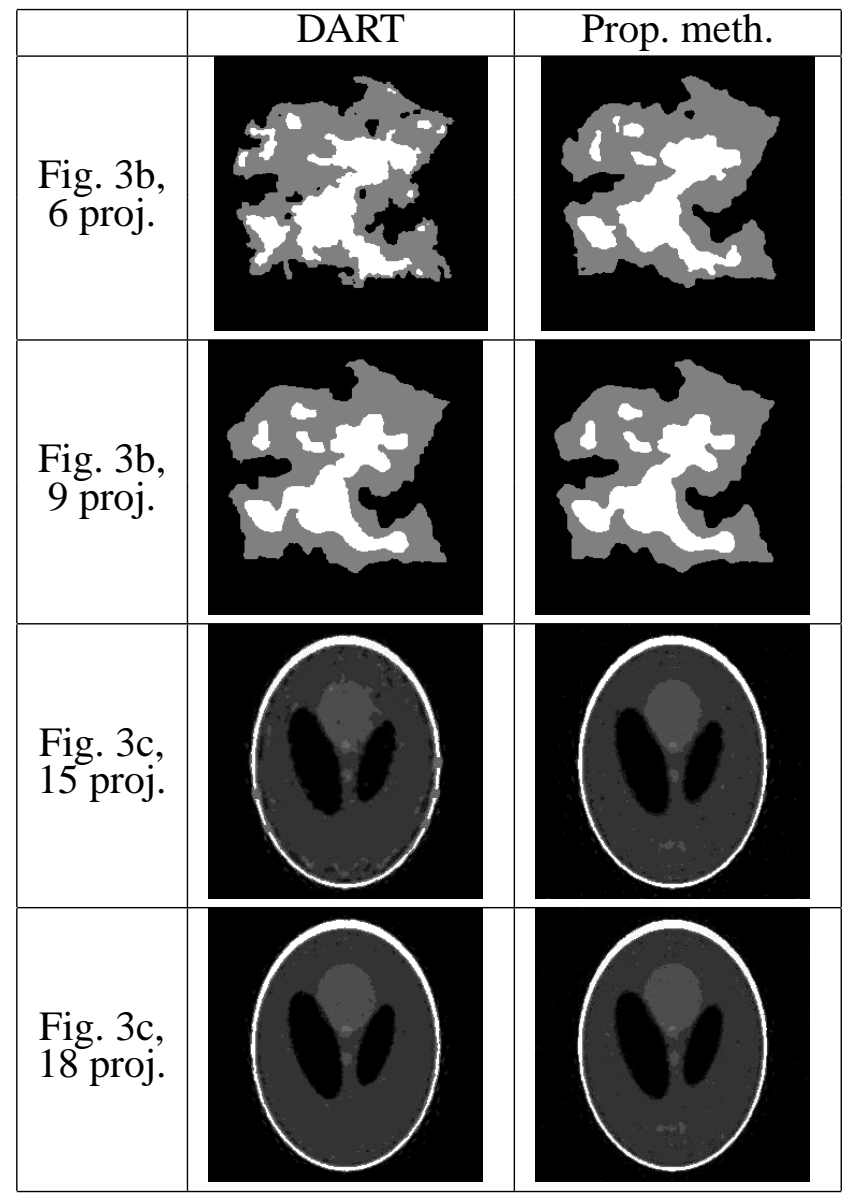

Figure 5: Sample of reconstructions of multivalued phantoms of Figure $3 \mathrm{~b}-\mathrm{c}$, produced by the DART and our proposed algorithm, from different number of projections. 
Table 1: Reconstruction errors and computation times of the compared algorithms, reconstructing the phantoms of Figure 3. The error measurement is computed by (10), and the computational time is given in seconds. Reconstructions of the DC algorithm could only be performed on binary test images. In each row, the best result is highlighted in bold.

\begin{tabular}{|c|c|c|c|c|c|c|}
\hline \multicolumn{7}{|c|}{ Figure $3 a$} \\
\hline & \multicolumn{2}{|c|}{$\overline{\mathrm{DC}}$} & \multicolumn{2}{|c|}{ DART } & \multicolumn{2}{|c|}{ Proposed method } \\
\hline P. Num. & Error & Time & Error & Time & Error & Time \\
\hline 2 & $90.7 \%$ & $12.1 \mathrm{~s}$ & $85.6 \%$ & $6.6 \mathrm{~s}$ & $107.4 \%$ & $10.1 \mathrm{~s}$ \\
\hline 3 & $22.0 \%$ & $12.4 \mathrm{~s}$ & $52.9 \%$ & $5.4 \mathrm{~s}$ & $30.8 \%$ & $11.2 \mathrm{~s}$ \\
\hline 4 & $1.2 \%$ & $13.6 \mathrm{~s}$ & $44.9 \%$ & $8.0 \mathrm{~s}$ & $22.4 \%$ & $11.8 \mathrm{~s}$ \\
\hline 5 & $0.3 \%$ & $12.5 \mathrm{~s}$ & $29.9 \%$ & $9.5 \mathrm{~s}$ & $7.9 \%$ & $12.7 \mathrm{~s}$ \\
\hline 6 & $0.2 \%$ & $8.1 \mathrm{~s}$ & $0.2 \%$ & $2.7 \mathrm{~s}$ & $0.8 \%$ & $7.6 \mathrm{~s}$ \\
\hline 9 & $0.2 \%$ & $6.5 \mathrm{~s}$ & $0.0 \%$ & $0.8 \mathrm{~s}$ & $0.3 \%$ & $4.6 \mathrm{~s}$ \\
\hline 12 & $0.0 \%$ & $7.2 \mathrm{~s}$ & $0.0 \%$ & $0.9 \mathrm{~s}$ & $0.1 \%$ & $4.8 \mathrm{~s}$ \\
\hline 15 & $0.0 \%$ & $8.7 \mathrm{~s}$ & $\mathbf{0 . 0 \%}$ & $1.2 \mathrm{~s}$ & $0.1 \%$ & $5.8 \mathrm{~s}$ \\
\hline 18 & $0.0 \%$ & $8.7 \mathrm{~s}$ & $0.0 \%$ & $0.9 \mathrm{~s}$ & $0.1 \%$ & $5.8 \mathrm{~s}$ \\
\hline \multicolumn{7}{|c|}{ Figure 3b } \\
\hline & \multicolumn{2}{|c|}{$\mathrm{DC}$} & \multicolumn{2}{|c|}{ DART } & \multicolumn{2}{|c|}{ Prop. meth. } \\
\hline P. Num. & Error & Time & Error & Time & Error & Time \\
\hline 2 & - & - & $62.9 \%$ & $6.7 \mathrm{~s}$ & $52.7 \%$ & $10.4 \mathrm{~s}$ \\
\hline 3 & - & - & $45.1 \%$ & $8.0 \mathrm{~s}$ & $41.9 \%$ & $11.4 \mathrm{~s}$ \\
\hline 4 & - & - & $43.4 \%$ & $8.6 \mathrm{~s}$ & $35.4 \%$ & $12.2 \mathrm{~s}$ \\
\hline 5 & - & - & $36.4 \%$ & $9.4 \mathrm{~s}$ & $26.4 \%$ & $13.2 \mathrm{~s}$ \\
\hline 6 & - & - & $27.0 \%$ & $10.2 \mathrm{~s}$ & $11.6 \%$ & $13.8 \mathrm{~s}$ \\
\hline 9 & - & - & $0.7 \%$ & $4.5 \mathrm{~s}$ & $1.9 \%$ & $15.6 \mathrm{~s}$ \\
\hline 12 & - & - & $0.4 \%$ & $14.9 \mathrm{~s}$ & $1.0 \%$ & $11.6 \mathrm{~s}$ \\
\hline 15 & - & - & $0.3 \%$ & $2.3 \mathrm{~s}$ & $0.8 \%$ & $11.6 \mathrm{~s}$ \\
\hline 18 & - & - & $0.1 \%$ & $21.3 \mathrm{~s}$ & $0.6 \%$ & $10.9 \mathrm{~s}$ \\
\hline \multicolumn{7}{|c|}{ Figure 3c } \\
\hline & \multicolumn{2}{|c|}{$\mathrm{DC}$} & \multicolumn{2}{|c|}{ DART } & \multicolumn{2}{|c|}{ Prop. meth. } \\
\hline P. Num. & Error & Time & Error & Time & Error & Time \\
\hline 2 & - & - & $84.4 \%$ & $6.7 \mathrm{~s}$ & $85.7 \%$ & $9.3 \mathrm{~s}$ \\
\hline 3 & - & - & $77.3 \%$ & $8.2 \mathrm{~s}$ & $82.5 \%$ & $6.0 \mathrm{~s}$ \\
\hline 4 & - & - & $75.3 \%$ & $8.8 \mathrm{~s}$ & $81.0 \%$ & $8.0 \mathrm{~s}$ \\
\hline 5 & - & - & $73.3 \%$ & $9.7 \mathrm{~s}$ & $74.2 \%$ & $10.2 \mathrm{~s}$ \\
\hline 6 & - & - & $74.1 \%$ & $10.2 \mathrm{~s}$ & $70.0 \%$ & $12.7 \mathrm{~s}$ \\
\hline 9 & - & - & $57.0 \%$ & $12.6 \mathrm{~s}$ & $46.8 \%$ & $14.7 \mathrm{~s}$ \\
\hline 12 & - & - & $33.9 \%$ & $14.5 \mathrm{~s}$ & $24.8 \%$ & $11.4 \mathrm{~s}$ \\
\hline 15 & - & - & $22.0 \%$ & $18.0 \mathrm{~s}$ & $16.3 \%$ & $8.6 \mathrm{~s}$ \\
\hline 18 & - & - & $15.7 \%$ & $20.8 \mathrm{~s}$ & $14.0 \%$ & $8.0 \mathrm{~s}$ \\
\hline
\end{tabular}

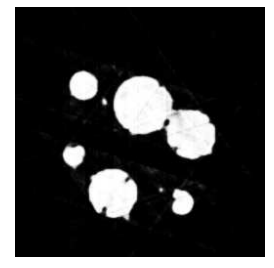

a)

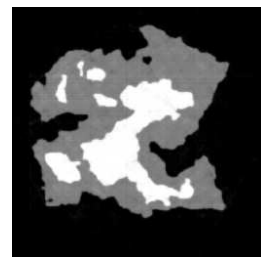

b)

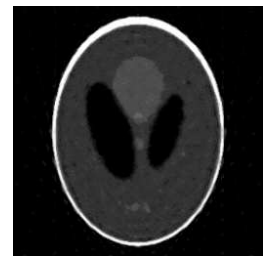

c)
Figure 6: Continuous results of the proposed reconstruction algorithm, without the final thresholding. (The images a), b) and c) were reconstructed from 5, 6 , and 15 projections, respectively.)
Starting to increase the number of projections, the amount of information in the data was also increasing and the results provided by the algorithms began to improve as well. The optimization based algorithms (DC and the proposed method) showed a faster improvement with the increasing of the projection numbers, therefore after a certain number of projections they started to give better results than the DART. Usually, the advantage of the optimization-based methods caused a sudden drop in the reconstruction error, when the algorithms started to give more accurate results. Thus, we can deduce that these two algorithms can ensure more or less accurate reconstructions from fewer projections than the DART.

Later, when we had even more projections with more than sufficient information for an accurate reconstruction, again the DART provided the best reconstructions, by producing slightly better results than the other two methods.

When comparing the energy minimization based methods, we can observe that on binary images the DC algorithm works better than our proposed method. This might be due to the form of the discretization term in the energy function. The DC algorithm is specialized for binary tomography, and aims a full binarization in the optimization process. The drawback is that the original DC algorithm is not capable of performing multivalued tomography at all.

On the other hand, our algorithm needs a different approach for having the generality to be able to reconstruct multivalued images, and it only makes an approximate discretization. This means that in a later state of the energy minimization process - without the final thresholding - we get a semi-discrete, semicontinuous result. This intermediate result is produced by taking into account that we are looking for a discrete solution, but still contains some uncertainty of the values (some of the examples of such results can be seen in Fig. 6). This kind of soft discretization is necessary for the multivalued reconstruction in our method, but it reduces the accuracy of the algorithm on binary images.

Finally, regarding the computational time of the algorithms, we found that depending on the conditions of the reconstruction and the image processed, one or another algorithm gave results faster than the other ones. Still, in general the time requirements showed to be similar.

In summary, the performance of the algorithms were similar on our dataset. All three methods can yield highly accurate reconstructions. Nevertheless, we found that the energy minimization-based methods gave slightly better results when the reconstructions were performed from a low number of projections, but the results of DART were better with more projections. This diversity makes all the algorithms 
valuable, and in a practical application, we would advise to choose from them based on the conditions of the reconstruction.

\section{Conclusion and Further Work}

In this paper we proposed a new algorithm for multivalued discrete tomography, that is based on the minimization of a suitably constructed energy function. We compared our method to two existing reconstruction algorithms by performing experimental tests on a set of software phantoms. Our results show that the proposed method performs better than the other ones under certain conditions, thus it should be considered a useful alternative for discrete tomography reconstruction.

Also, neglecting the final thresholding step of our algorithm, one can gain reconstruction results which - in some way - might describe the uncertainty in the reconstruction. We think that this property is worth to be investigated in more detail.

In our future work we intend to improve the algorithm by modifying the minimized energy function, and to study the applicability of the technique in different practical fields of discrete tomography. Also, we will make efforts to prove the convergence of the method.

\section{REFERENCES}

[1] P. Balázs, M. Gara, An evolutionary approach for object-based image reconstruction using learnt priors, Lecture Notes in Computer Science vol. 5575, pp. 520-529 (2009).

[2] K.J. Batenburg, An evolutionary algorithm for discrete tomography, Disc. Appl. Math. 151, pp. 36-54 (2005).

[3] K.J. Batenburg, J. Sijbers, DART: a fast heuristic algebraic reconstruction algorithm for discrete tomography, IEEE Conference on Image Processing IV, pp. 133-136 (2007).

[4] K.J. Batenburg, J. Sijbers, DART: a practical reconstruction algorithm for discrete tomography, IEEE Transactions on Image Processing 20(9), pp. 2542-2553 (2011).

[5] Brunetti, S., Del Lungo, A., Del Ristoro, F., Kuba, A. and Nivat, M. Reconstruction of 4- and 8-connected convex discrete sets from row and column projections, Lin. Alg. Appl. 339, pp. 37-57 (2001).

[6] J. Baumann, Z. Kiss, S. Krimmel, A. Kuba, A. Nagy, L. Rodek, B. Schillinger, J. Stephan, Discrete Tomography Methods for Nondestructive Testing, Chapter 14 of (11).

[7] M. Chrobak, Ch. Dürr, Reconstructing hvconvex polyominoes from orthogonal pro- jections. Information Processing Letters 69(6), pp. 283-289 (1999).

[8] V. Di Gesù, G. Lo Bosco, F. Millonzi, C. Valenti, A memetic algorithm for binary image reconstruction, Lecture Notes in Computer Science vol. 4958, pp. 384-395 (2008).

[9] R. J. Gardner, P. Gritzmann, Discrete tomography: Determination of finite sets by $X$-rays, Trans. Amer. Math. Soc. 349(6), pp. 22712295 (1997).

[10] G.T. Herman, A. Kuba (Eds.), Discrete Tomography: Foundations, Algorithms and Applications, Birkhäuser, Boston, 1999.

[11] G.T. Herman, A. Kuba (Eds.), Advances in Discrete Tomography and Its Applications, Birkhäuser, Boston, 2007.

[12] A. C. Kak, M. Slaney, Principles of computerized tomographic imaging, IEEE Press, New York, 1999.

[13] T. Lukić, Discrete tomography reconstruction based on the multi-well potential, Lecture Notes in Computer Science vol. 6636, pp. 335-345 (2011).

[14] A. Nagy, A. Kuba, Reconstruction of binary matrices from fan-beam projections, Acta $\mathrm{Cy}-$ bernetica 17(2), pp. 359-385 (2005).

[15] T. Schüle, C. Schnörr, S. Weber, J. Hornegger, Discrete tomography by convex-concave regularization and D.C. programming, Discrete Applied Mathematics 151, pp. 229243 (2005).

[16] T. Schüle, S. Weber, C. Schnörr, Adaptive reconstruction of discrete-valued objects from few projections, Electronic Notes in Discrete Mathematics vol. 20, pp. 365-384 (2005).

[17] L. Varga, P. Balázs, A. Nagy, Directiondependency of binary tomographic reconstruction algorithms, Graphical Models 73(6), pp. 365-375 (2011).

[18] S. Weber, Discrete tomography by convex concave regularization using linear and quadratic optimization, $\mathrm{PhD}$ thesis, Heidelberg University, 2009. 